\title{
Performance analysis for time-frequency MUSIC algorithm in presence of both additive noise and array calibration errors
}

\author{
Mohamed Khodja ${ }^{1 *}$, Adel Belouchrani ${ }^{1}$ and Karim Abed-Meraim²
}

\begin{abstract}
This article deals with the application of Spatial Time-Frequency Distribution (STFD) to the direction finding problem using the Multiple Signal Classification (MUSIC)algorithm. A comparative performance analysis is performed for the method under consideration with respect to that using data covariance matrix when the received array signals are subject to calibration errors in a non-stationary environment. An unified analytical expression of the Direction Of Arrival (DOA) error estimation is derived for both methods. Numerical results show the effect of the parameters intervening in the derived expression on the algorithm performance. It is particularly observed that for low Signal to Noise Ratio (SNR) and high Signal to sensor Perturbation Ratio (SPR) the STFD method gives better performance, while for high SNR and for the same SPR both methods give similar performance.
\end{abstract}

\section{Introduction}

Advances in antennas technology and signal processing have allowed the emergence of the generation of "the so-called" smart antennas. The latter are commonly used for the direction of arrival (DOA) estimation of far field sources from multiple antenna outputs. DOA estimation is currently one of the important issues in next generation wireless communications, namely the space division multiple access (SDMA).

The techniques used in DOA estimation depend on the nature of the signals under consideration. When the impinging signals are stationary, conventional methods such as the eigen-subspace decomposition of the covariance data matrix are usually used $[1,2]$. These methods lose of their performance for non-stationary signals. Other high resolution techniques like the ones based on the spatial time-frequency distributions (STFD) were introduced to cope with the non-stationary nature of the signals [3-6]. STFD based methods are based on the use of the quadratic time-frequency distributions of the received signals at the array antenna. High resolution DOA estimation consists of applying the eigen-subspace

\footnotetext{
* Correspondence: mohamed.khodja@enp.edu.dz

${ }^{1}$ Electrical Engineering Department, Ecole Nationale Polytechnique, Algiers, Algeria

Full list of author information is available at the end of the article
}

decomposition of the STFD instead of the conventional covariance data matrix. In [7], performance comparison between the Time-Frequency MUSIC (TF-MUSIC) and the conventional MUSIC, in presence of additive noise, is provided. In [8], statistical performance analysis in presence of sensor errors without considering the presence of observation noise are conducted for DOA estimation algorithms based on second-order statistics (SOS). In [9], first-order perturbation analysis of the conventional SOS MUSIC and root-MUSIC algorithms is presented.

In this article, our interest is focused on the performance analysis of the conventional MUSIC and the TFMUSIC algorithms in the presence of both additive noise and sensor errors. These errors can incorporate the effect of imprecisely known sensor location, perturbations in the antenna amplitude and phase patterns that we consider as the calibration errors. A unified analytical expression of the DOA error estimation is derived for both methods. An analysis of the effect of the sensor perturbations on the performance of the considered algorithms is also provided.

\section{Notations}

In this article, boldface symbols are used in lower-case letters for vectors (e.g., a), and in upper-case letters for 
matrices (e.g., A). The principal symbols and notations used are listed below.

$K$ number of signal sources

$L \quad$ number of sensors

$M \quad$ number of snapshots

$\theta_{k} \quad k$ th direction of arrival

$\mathbf{a}\left(\theta_{k}\right) \quad k$ th steering vector

$\mathbf{A}(\theta)$ array response matrix

I $L \times L$ identity matrix

(.)" complex conjugate of (.)

$(.)^{H} \quad$ complex conjugate transpose of (.)

$\operatorname{Tr}($.$) \quad trace of (.)$

$\delta_{i, k} \quad$ Kronecker delta function.

\section{Data model}

We consider a uniform linear array (ULA) of $L$ sensors receiving $K$ incident signals. The data vector at the output of the sensors at time $t$ is given by,

$$
\mathbf{y}(t)=\mathbf{A}(\theta) \mathbf{s}(t)+\mathbf{n}(t)
$$

This model is commonly used in array signal processing, where $\mathbf{y}(t)=\left[\begin{array}{lll}y_{1}(t) & \ldots & y_{L}(t)\end{array}\right]^{T}$ is the output array vector, with $y_{i}(t), i=1, \ldots, L$, the output of the $i$ th sensor. A $(\theta)=\left[\begin{array}{lll}\mathbf{a}\left(\theta_{1}\right) & \ldots & \mathbf{a}\left(\theta_{K}\right)\end{array}\right]$ is the $L \times K$ structured mixing matrix known as the array steering matrix, each vector $\mathbf{a}\left(\theta_{j}\right), j=1, \ldots, K$, is an array response to a signal $s_{j}(t)$ from direction $\theta_{j} . \mathbf{n}(t)=\left[n_{1}(t) \ldots n_{L}(t)\right]^{T}$ is an additive noise that we assume to be a zero mean white complex stationary process with covariance matrix $\mathbf{R}_{\mathbf{n n}}=E\left[n(t) n^{H}(t)\right]=\sigma_{n}^{2} \mathbf{I}$, and $\mathbf{s}(t)=\left[s_{1}(t) \ldots s_{K}(t)\right]^{T}$ is the source signal vector with covariance matrix $\mathbf{R}_{\mathbf{s s}}=E$ $\left[\mathbf{s}(t) \mathbf{s}^{H}(t)\right]$. The source signals $s_{i}(t), i=1, \ldots, K$, are assumed mutually uncorrelated and frequency modulated signals of the form

$$
s_{i}(t)=S_{i} e^{j \varphi_{i}(t)}
$$

where $S_{i}$ and $\phi_{i}(t)$ are the amplitude and phase of the $i$ th source signal. The amplitude $S_{i}$ is assumed to be a random variable with zero mean and variance $\sigma_{s_{i}}^{2}$, while the phase $\phi_{i}(t)$ is time varying.

When the sensors are subject to perturbations due to errors in calibration or in sensor location, the signal response is corrupted by errors characterized by the vectors $\left\{\Delta \mathbf{a}_{i}\right\}_{i=1}^{K}$ added to the steering vectors $\left\{\mathbf{a}\left(\theta_{i}\right)\right\}_{i=1}^{K}$. Theses errors are randomly changing from one observation period to another. Furthermore, we assume that these errors are uncorrelated random variables with zero mean and equal variances $\sigma_{\Delta a}^{2}$. The disturbed array manifold matrix can then be written as,

$$
\tilde{\mathbf{A}}(\theta)=\mathbf{A}(\theta)+\Delta \mathbf{A}
$$

This is a widely used disturbance model [8-12] that take into account different sensor errors including those related to calibration. According to Equations (1) and (3), the disturbed data model is,

$$
\tilde{\mathbf{y}}(t)=[\mathbf{A}(\theta)+\Delta \mathbf{A}] \mathbf{s}(t)+\mathbf{n}(t)
$$

and can be expressed under the form

$$
\tilde{\mathbf{y}}(t)=\stackrel{\circ}{\mathbf{y}}(t)+\mathbf{p}(t)
$$

where $\stackrel{\circ}{\mathbf{y}}(t)$ is the perturbation free data matrix, and $\mathbf{p}$ $(t)$ is the perturbation vector including the sensor errors and the additive noise assumed uncorrelated,

$$
\begin{aligned}
& \stackrel{\circ}{\mathbf{y}}(t)=\mathbf{A}(\theta) \mathbf{s}(t) \\
& \mathbf{p}(t)=\Delta \operatorname{As}(t)+\mathbf{n}(t)
\end{aligned}
$$

\section{Covariance matrix perturbation}

The covariance matrix of (5) is given by,

$$
\begin{aligned}
\mathbf{R}_{\tilde{\mathbf{y}} \tilde{\mathbf{y}}} & =E\left[\tilde{\mathbf{y}}(t) \tilde{\mathbf{y}}^{H}(t)\right] \\
& =E\left[(\stackrel{\circ}{\mathbf{y}}(t)+\mathbf{p}(t))\left(\stackrel{\circ}{\mathbf{y}}^{H}(t)+\mathbf{p}^{H}(t)\right)\right]
\end{aligned}
$$

Substituting $\mathbf{p}(t)$ with its expression (7) into (8), and since $\Delta \mathbf{A}$ is zero mean $\left(E[\Delta \mathbf{A}]=E\left[\Delta \mathbf{A}^{H}\right]=0\right)$, it results that,

$$
\mathbf{R}_{\tilde{\mathbf{y}} \tilde{\mathbf{y}}}=E[\stackrel{\circ}{\mathbf{y}}(t) \stackrel{\circ}{\stackrel{\mathrm{y}}{H}}(t)]+E\left[\Delta \mathbf{A} \mathbf{s}(t) \mathbf{s}^{H}(t) \Delta \mathbf{A}^{H}\right]+\sigma_{n}^{2} \mathbf{I}
$$

where $\mathbf{I}$ is the $L \times L$ identity matrix, and,

$$
\begin{aligned}
E\left[\stackrel{\circ}{\mathbf{y}}(t) \stackrel{\circ}{\mathbf{y}}^{H}(t)\right] & =\mathbf{A}(\theta) E\left[\mathbf{s}(t) \mathbf{s}^{H}(t)\right] \mathbf{A}^{H}(\theta) \\
& =\mathbf{A}(\theta) \mathbf{R}_{\mathbf{s s}} \mathbf{A}^{H}(\theta)
\end{aligned}
$$

where $\mathbf{R}_{\mathbf{s s}}=E\left[\mathbf{s}(t) \mathbf{s}^{H}(t)\right]=\operatorname{diag}\left[\sigma_{s_{1}}^{2}, \ldots, \sigma_{s_{K}}^{2}\right]$. It is proven in Appendix 1 that the second term in the Equation (9) is given by,

$$
E\left[\Delta \mathbf{A s}(t) \mathbf{s}^{H}(t) \Delta \mathbf{A}^{H}\right]=\sigma_{\Delta \mathbf{a}}^{2} \operatorname{Tr}\left(\mathbf{R}_{\mathbf{s s}}\right) \mathbf{I}
$$

where $\operatorname{Tr}\left(\mathbf{R}_{\mathbf{s s}}\right)=\sum_{i=1}^{K} \sigma_{s_{i}}^{2}$. Substituting (10) and

into (9), the covariance matrix of the perturbed data can then be written as,

$$
\mathbf{R}_{\tilde{\mathbf{y}} \tilde{\mathbf{y}}}=\mathbf{A}(\theta) \mathbf{R}_{\mathbf{s s}} \mathbf{A}^{H}(\theta)+\left[\sigma_{\Delta \mathbf{a}}^{2} \operatorname{Tr}\left(\mathbf{R}_{\mathbf{s s}}\right)+\sigma_{n}^{2}\right] \mathbf{I}
$$

\section{STFD matrix perturbation}

The STFD based techniques exploit both the time-frequency representation of the signals and the spatial 
diversity provided by the multi-sensor platform. In this article, we consider the discrete form of the spatial pseudo Wigner-Ville distribution (PWVD) matrix using a rectangular window of odd length $N$ that we apply to the perturbed data $\tilde{\mathbf{y}}(t)$ vector,

$$
\mathbf{D}_{\tilde{\mathbf{y}} \tilde{\mathbf{y}}}(t, f)=\sum_{\tau=-(N-1) / 2}^{(N-1) / 2} \tilde{\mathbf{y}}(t+\tau) \tilde{\mathbf{y}}^{H}(t-\tau) e^{-j 4 \pi f \tau}
$$

Substituting (5) into (13) we obtain,

$$
\mathbf{D}_{\tilde{\mathbf{y}} \tilde{\mathbf{y}}}(t, f)=\mathbf{D}_{\mathbf{y} \mathbf{y}}(t, f)+\mathbf{D}_{\mathbf{y} \mathbf{p}}(t, f)+\mathbf{D}_{\mathbf{p y}}(t, f)+\mathbf{D}_{\mathbf{p p}}(t, f)
$$

$\mathbf{D}_{\mathbf{y y}} \circ(t, f)$ is the STFD data matrix in absence of the sensor error and the additive noise, and $\mathbf{D}_{\mathbf{p p}}$ is the perturbation STFD matrix, while $\mathbf{D}_{\mathbf{y} \mathbf{p}}$ and $\mathbf{D}_{\mathbf{p y}}$ are the cross STFDs matrices. Under the property of zero mean of noise $\mathbf{n}(t)$ and sensor error represented by $\Delta \mathbf{A}$, the expectation of the cross-terms vanishes (i,e., $E\left[\mathbf{D}_{\mathbf{y p}}\right]=0$ and $\left.E\left[\mathbf{D}_{\mathrm{py}}^{\circ}\right]=0\right)$, and it follows,

$$
E\left[\mathbf{D}_{\tilde{\mathbf{y}} \mathbf{y}}(t, f)\right]=E\left[\mathbf{D}_{\mathbf{y} \mathbf{y}}(t, f)\right]+E\left[\mathbf{D}_{\mathbf{p p}}(t, f)\right]
$$

Considering the spatially and temporally white assumptions of the perturbed steering matrix and noise, and using Equations (6) and (7) the above equation becomes,

$$
E\left[\mathbf{D}_{\tilde{\mathbf{y}}}(t, f)\right]=E\left[\mathbf{D}_{\tilde{y} \mathbf{y}}(t, f)\right]+E\left[\Delta \mathbf{A D}_{\mathbf{s s}}(t, f) \Delta \mathbf{A}^{H}\right]+E\left[\mathbf{D}_{\mathbf{n n}}(t, f)\right]
$$

Where

$$
E\left[\mathbf{D}_{\mathbf{n n}}(t, f)\right]=\sigma_{n}^{2} \mathbf{I}
$$

and

$$
\mathbf{D}_{\mathbf{y} \mathbf{y}}(t, f)=\mathbf{A}(\theta) \mathbf{D}_{\mathbf{s s}}(t, f) \mathbf{A}(\theta)^{H}
$$

$\mathbf{D}_{\mathbf{s s}}(t, f)$ is a $K \times K$ source signal STFD matrix whose elements are given by,

$$
d_{S_{i_{S_{k}}}}(t, f)=S_{i} S_{k} \sum_{\tau=-(N-1) / 2}^{(N-1) / 2} e^{j\left[\varphi_{i}(t+\tau)-\varphi_{k}(t-\tau)-4 \pi f \tau\right]}
$$

where the diagonal elements $d_{s_{i} s_{i}}(t, f)$ are the autoTFDs of the source signals, while the off-diagonal elements $d_{s_{i} s_{k}}(t, f),(i \neq k)$, are the cross-TFDs. We consider only the t-f points along the actual instantaneous frequency (IF) of each signal. Furthermore, assuming a second-order approximation of the derivative of the phase, we have,

$$
\varphi_{i}(t+\tau)-\varphi_{i}(t-\tau)-4 \pi f_{i}(t) \tau=0
$$

where the $\operatorname{IF} f_{i}(t)$ is given by,

$$
f_{i}(t)=\frac{1}{2 \pi} \frac{d \varphi_{i}(t)}{d t}
$$

Therefore, it results from (19) that,

$$
E\left[d_{s_{i} s_{i}}(t, f)\right]=N \sigma_{s_{i}}^{2}
$$

In order to exploit the STFD given by (13) under an eigen-decomposition form, we use an averaging method which consists of averaging the STFD matrix $\mathbf{D}_{\tilde{y} \tilde{y}}(t, f)$ at $\left(t_{i}, f_{i}\right)$ points over the selected sources and over a number of $T_{o}$ selected tf-points $\left(T_{o}=M-N+1\right)$, where $M$ is the number of snapshots.

$$
\overline{\mathbf{D}}_{\tilde{\mathbf{y}} \tilde{\mathbf{y}}}=\frac{1}{K T_{o}} \sum_{k=1}^{K} \sum_{i=1}^{T_{o}} \mathbf{D}_{\tilde{\mathbf{y}} \tilde{y}}\left(t_{i}, f_{k, i}\right)
$$

whose expectation is given by,

$$
E\left[\overline{\mathbf{D}}_{\tilde{\mathbf{y}}}\right]=\frac{1}{K T_{o}} \sum_{k=1}^{K} \sum_{i=1}^{T_{o}} E\left[\mathbf{D}_{\tilde{y} \tilde{y}}\left(t_{i}, f_{k, i}\right)\right]
$$

where $f_{k, i}$ is the IF of the $k$ th signal at the $i$ th time sample. Considering the Equation (14) for the $\left(t_{i}, f_{k, i}\right)$ points and substituting it into (23), we obtain, after a straightforward calculation carried out in Appendix 2, the following expression,

$$
\hat{\mathbf{D}}_{\tilde{y} \tilde{y}}=E\left[\overline{\mathbf{D}}_{\tilde{\mathbf{y}} \tilde{y}}\right]=\frac{N}{K} \mathbf{A}(\theta) \mathbf{R}_{\mathbf{s s}} \mathbf{A}^{H}(\theta)+\left[\frac{N}{K} \sigma_{\Delta a}^{2} \operatorname{Tr}\left(\mathbf{R}_{\mathbf{s s}}\right)+\sigma_{n}^{2}\right] \mathbf{I}
$$

\section{Perturbation analysis: unified formulation}

Expressions of $\mathbf{R}_{\tilde{\mathbf{y}} \tilde{y}}$ and $\hat{\mathbf{D}}_{\tilde{\mathbf{y}} \tilde{\mathbf{y}}}$ given by (12) and (25), respectively, are a sum of three terms corresponding to the perturbation free signal, the sensor error, and the additive noise. These expressions can be written under a unified form as follows,

$$
\mathbf{P}_{\mathbf{y} \tilde{y}}=\mathbf{P}_{\mathbf{y} \mathbf{y}}+\sigma_{p}^{2} \mathbf{I}
$$

where,

$$
\mathbf{P}_{\mathrm{yy}}=\left\{\begin{array}{l}
\mathbf{R}_{\mathrm{yy}}=\mathbf{A}(\theta) \mathbf{R}_{\mathrm{ss}} \mathbf{A}^{H}(\theta), \quad \text { for covariance based method, } \\
\hat{\mathbf{D}}_{\mathrm{y} \grave{y}}=\frac{N}{K} \mathbf{A}(\theta) \mathbf{R}_{\mathrm{ss}} \mathbf{A}^{H}(\theta), \text { for STFD based method }
\end{array}\right.
$$

$\hat{\mathbf{D}}_{\mathbf{y}}$ i is the STFD matrix corresponding to the sources in absence of both noise and sensor errors, and,

$$
\sigma_{p}^{2}=\left\{\begin{array}{l}
\left(\sigma_{p}^{o s s}\right)^{2}=\sigma_{\Delta a}^{2} \operatorname{Tr}\left(\mathbf{R}_{\mathrm{ss}}\right)+\sigma_{n}^{2}, \quad \text { for covariance based method, } \\
\left(\sigma_{p}^{\prime f}\right)^{2}=\frac{N}{K} \sigma_{\Delta a}^{2} \operatorname{Tr}\left(\mathbf{R}_{\mathrm{ss}}\right)+\sigma_{n}^{2}, \text { for STFD based method }
\end{array}\right.
$$


In Equation (28), the superscript "sos" stands for second-order statistics associated to the covariance matrix based method, and the superscript " $t f^{\text {" }}$ stands for timefrequency associated to the STFD based method.

\section{Remarks}

- It is important to observe from Equation (25) that in absence of additive noise, the STFD based method do not make any improvement compared to the conventional MUSIC.

- On the other hand, in presence of additive noise, as it is always the case in a real-life situation, the signal to noise ratio (SNR) is improved by a factor $N / K$ for the STFD based method. This improvement would be still better for larger window length.

According to (26) and (27), $\mathbf{P}_{\tilde{y} \tilde{y}}$ is a positive definite matrix and can be expressed under an eigen-decomposition form as follows,

$$
\mathbf{P}_{\tilde{\mathbf{y}} \tilde{y}}=\left\{\begin{array}{l}
\tilde{\mathbf{U}}^{\text {sos }} \tilde{\boldsymbol{\Lambda}}^{\text {sos }}\left(\tilde{\mathbf{U}}^{\text {sos }}\right)^{H}, \text { for covariance based method, } \\
\tilde{\mathbf{U}}^{t f} \tilde{\boldsymbol{\Lambda}}^{t f}\left(\tilde{\mathbf{U}}^{t f}\right)^{H}, \quad \text { for STFD based method }
\end{array}\right.
$$

which can be expressed under the following unified form

$$
\mathbf{P}_{\tilde{\mathbf{y}} \tilde{\mathbf{y}}}=\sum_{i=1}^{L} \lambda_{i} \tilde{\mathbf{u}}_{i} \tilde{\mathbf{u}}_{i}^{H}=\tilde{\mathbf{U}} \tilde{\mathbf{\Lambda}} \tilde{\mathbf{U}}^{H}
$$

where $\tilde{\boldsymbol{\Lambda}}=\operatorname{diag}\left[\lambda_{i}, i=1, \ldots, L\right]$ is the matrix of the eigenvalues of $\mathbf{P}_{\tilde{\mathbf{y}} \tilde{\mathbf{y}}}$, and $\left\{\tilde{\mathbf{u}}_{i}\right\}_{i=1}^{L}$ are the corresponding eigenvectors.

These definitions and notations being made, and for the sake of simplicity we consider in the sequel only the unified form $P_{\tilde{y} \tilde{y}}$ given by Equation (30) to deal with both the covariance and the STFD based methods.

The matrix $\tilde{\mathbf{U}}$ can be arranged as $\tilde{\mathbf{U}}=\left[\begin{array}{ll}\tilde{\mathbf{U}}_{s} & \tilde{\mathbf{U}}_{p}\end{array}\right]$, where $\tilde{\mathbf{U}}_{s}=\left[\tilde{\mathbf{u}}_{1}, \ldots, \tilde{\mathbf{u}}_{K}\right]$ forms the signal subspace, and $\tilde{\mathbf{U}}_{p}=\left[\tilde{\mathbf{u}}_{K+1}, \ldots, \tilde{\mathbf{u}}_{L}\right]$ forms the perturbed subspace known as the orthogonal subspace. The Equation (30) can then be rewritten as follows,

$$
\begin{aligned}
\mathbf{P}_{\tilde{\mathbf{y}} \tilde{y}} & =\left(\tilde{\mathbf{U}}_{s} \tilde{\mathbf{U}}_{p}\right)\left(\begin{array}{cc}
\tilde{\boldsymbol{\Lambda}}_{s} & 0 \\
0 & \tilde{\boldsymbol{\Lambda}}_{p}
\end{array}\right)\left(\begin{array}{c}
\tilde{\mathbf{U}}_{s}^{H} \\
\tilde{\mathbf{U}}_{p}^{H}
\end{array}\right) \\
& =\tilde{\mathbf{U}}_{s} \tilde{\boldsymbol{\Lambda}}_{s} \tilde{\mathbf{U}}_{s}^{H}+\tilde{\mathbf{U}}_{p} \tilde{\boldsymbol{\Lambda}}_{p} \tilde{\mathbf{U}}_{p}^{H}
\end{aligned}
$$

where $\tilde{\boldsymbol{\Lambda}}_{s}=\operatorname{diag}\left[\lambda_{i}, i=1, \ldots, K\right]$ is the signal eigenvalue matrix, and $\tilde{\boldsymbol{\Lambda}}_{p}=\operatorname{diag}\left[\lambda_{i}, i=K+1, \ldots, L\right]$ the perturbation eigenvalue matrix. Thus, consequently to (31), a perturbation $\mathbf{P}_{\tilde{y} \tilde{y}}$ leads to the perturbation of both subspaces. The matrices $\tilde{\mathbf{U}}_{s}$ and $\tilde{\mathbf{U}}_{p}$ can then be expressed as,

$$
\tilde{\mathbf{U}}_{s}=\mathbf{U}_{s}+\Delta \mathbf{U}_{s} \quad \text { and } \quad \tilde{\mathbf{U}}_{p}=\mathbf{U}_{p}+\Delta \mathbf{U}_{p}
$$

where $\Delta \mathbf{U}_{s}$ and $\Delta \mathbf{U}_{p}$ represent, respectively, the perturbation of the signal subspace and the orthogonal subspace.

It is shown in Appendix 3 that the first-order expressions for $\Delta \mathbf{U}_{s}$ and $\Delta \mathbf{U}_{p}$ are given by,

$$
\begin{aligned}
& \Delta \mathbf{U}_{s}=\mathbf{U}_{p}+\mathbf{U}_{p}^{H} \Delta \mathbf{P}_{\mathbf{y y}} \mathbf{U}_{s} \Lambda_{s}^{-1} \\
& \Delta \mathbf{U}_{p}=-\mathbf{U}_{s} \Lambda_{s}^{-1} \mathbf{U}_{s}^{H} \Delta \mathbf{P}_{\mathbf{y y}}^{H} \mathbf{U}_{p}
\end{aligned}
$$

$\Delta \mathbf{P}_{\mathbf{y y}}$ represents the perturbation resulting from the presence of sensor errors and the additive noise in the data signal,

$$
\Delta \mathbf{P}_{\mathrm{yy}}=\left\{\begin{array}{l}
\Delta \mathbf{R}_{\mathrm{yy}}, \text { for covariance based method, } \\
\Delta \mathbf{D}_{\mathrm{yy}}, \text { for STFD based method }
\end{array}\right.
$$

where,

$$
\Delta \mathbf{R}_{\mathbf{y y}}=\frac{1}{M}\left[\tilde{\mathbf{y}}(t) \tilde{\mathbf{y}}(t)^{H}-\stackrel{\circ}{\mathbf{y}}(t) \stackrel{\circ}{\mathbf{y}}(t)^{H}\right]
$$

and,

$$
\Delta \mathbf{D}_{\mathbf{y y}}=\frac{1}{K T_{o}} \sum_{k=1}^{K} \sum_{i=1}^{T_{o}}\left[\mathbf{D}_{\tilde{y} \tilde{y}}\left(t_{i}, f_{k, i}\right)-\mathbf{D}_{\dot{y} \mathbf{y}}\left(t_{i}, f_{k, i}\right)\right]
$$

\section{DOA error estimation for the MUSIC and TF- MUSIC algorithms}

In the MUSIC and TF-MUSIC algorithms, the DOA are found by locating the $K$ largest peaks over the angle of arrival $\theta$ of the following spatial spectrum function,

$$
F(\theta)=\mathbf{a}(\theta)^{H} \tilde{\mathbf{U}}_{p} \tilde{\mathbf{U}}_{p}^{H} \mathbf{a}(\theta)
$$

In a perturbed environment, the direction of arrivals $\left\{\theta_{k}\right\}_{k=1}^{K}$ are corrupted by the errors $\left\{\Delta \theta_{k}\right\}_{k=1}^{K}$. Thus, the $k$ th estimated DOA corresponding to the $k$ th signal can be written as,

$$
\tilde{\theta}_{k}=\theta_{k}+\Delta \theta_{k}
$$

and the expression for the perturbation of DOA estimate, proved in Appendix 4, is given by,

$$
\Delta \theta_{k}=\frac{\operatorname{Re}\left[-\mathbf{a}\left(\theta_{k}\right)^{H} \Delta \mathbf{U}_{p} \mathbf{U}_{p}^{H} \mathbf{a}^{(1)}\left(\theta_{k}\right)\right]}{\left\|\mathbf{a}^{(1)}\left(\theta_{k}\right)^{H} \mathbf{U}_{p}\right\|^{2}}
$$




$$
\mathbf{a}^{(1)}\left(\theta_{k}\right)=\left.\frac{d \mathbf{a}(\theta)}{d \theta}\right|_{\theta=\theta_{k}}, k=1, \ldots, K \text {. Substituting } \Delta \mathbf{U}_{p} \text { by }
$$

its expression given in (34), we obtain,

$$
\Delta \theta_{k}=\frac{\operatorname{Re}\left[\mathbf{a}\left(\theta_{k}\right)^{H} \mathbf{U}_{s} \Lambda_{s}^{-1} \mathbf{U}_{s}^{H} \Delta \mathbf{P}_{\mathbf{y y}}^{H} \mathbf{U}_{p} \mathbf{U}_{p}^{H} \mathbf{a}^{(1)}\left(\theta_{k}\right)\right]}{\left\|\mathbf{a}^{(1)}\left(\theta_{k}\right)^{H} \mathbf{U}_{p}\right\|^{2}}
$$

which can be written under the following form [1],

$$
\Delta \theta_{k}=\frac{\operatorname{Re}\left[\alpha_{k}^{H} \Delta \mathbf{P}_{\mathrm{yy}}^{H} \beta_{k}\right]}{\gamma_{k}}
$$

where the vectors $\alpha_{k}$ and $\beta_{k}$ are given by,

$$
\begin{aligned}
& \alpha_{k}=\mathbf{U}_{s} \Lambda_{s}^{-1} \mathbf{U}_{s}^{H} \mathbf{a}\left(\theta_{k}\right) \\
& \beta_{k}=\mathbf{U}_{p} \mathbf{U}_{p}^{H} \mathbf{a}^{(1)}\left(\theta_{k}\right)
\end{aligned}
$$

and the scalar $\gamma_{k}$ is given by,

$$
\gamma_{k}=\left\|\mathbf{a}^{(1)}\left(\theta_{k}\right)^{H} \mathbf{U}_{p}\right\|^{2}
$$

The variance of the $k$ th DOA error estimate $\Delta \theta_{k}$ is then given by,

$$
\operatorname{var}\left(\Delta \theta_{k}\right)=\frac{1}{\gamma_{k}^{2}} \operatorname{var}\left[\operatorname{Re}\left(\alpha_{k}^{H} \Delta \mathbf{P}_{\mathbf{y y}}^{H} \beta_{k}\right)\right]
$$

Taking into account the notations given in the previous section, the results (41) and (46) are valid for both the conventional and time-frequency MUSIC.

$$
\operatorname{var}\left[\Delta \theta_{k}\right]=\left\{\begin{array}{l}
\operatorname{var}\left[\Delta \theta_{k}^{\text {sos }}\right], \text { for conventional MUSIC algorithm } \\
\operatorname{var}\left[\Delta \theta_{k}^{f]}\right], \text { for TF-MUSIC algorithm }
\end{array}\right.
$$

where,

$$
\operatorname{var}\left[\Delta \theta_{k}^{\text {sos }}\right]=\frac{1}{\left(\gamma_{k}^{\text {sos }}\right)^{2}} \operatorname{var}\left[\operatorname{Re}\left(\left(\alpha_{k}^{\text {sos }}\right)^{H} \Delta \mathbf{R}_{\mathrm{yy}}^{H} \beta_{k}^{\text {sos }}\right)\right]
$$

and

$$
\operatorname{var}\left[\Delta \theta_{k}^{t f}\right]=\frac{1}{\left(\gamma_{k}^{t f}\right)^{2}} \operatorname{var}\left[\operatorname{Re}\left(\left(\alpha_{k}^{t f}\right)^{H} \Delta \mathbf{D}_{\mathrm{yy}}^{H} \beta_{k}^{t f}\right)\right]
$$

\section{Performance evaluation}

In this section, simulation results are given for the STFD and the SOS MUSIC methods. Two received chirp source signals are considered, $s_{1}(t)=S_{1} \exp \left[j\left(\left(w_{12}\right.\right.\right.$ $\left.\left.\left.w_{11}\right)\left(t^{2} / 2\right)+w_{11} t\right)\right]$ and $s_{2}(t)=S_{2} \exp \left[j\left(\left(w_{22}-w_{21}\right)\left(t^{2} / 2\right)+\right.\right.$ $\left.w_{21} t\right)$ ] with powers $\sigma_{s_{1}}^{2}=1$ and $\sigma_{s_{2}}^{2}=4$, and with frequencies varying from $w_{11}=\pi / 6$ to $w_{12}=\pi$ and from $w_{21}=\pi$ to $w_{22}=\pi / 6$, respectively. The signals are positioned at angles $\theta_{1}=-10^{\circ}$ and $\theta_{2}=10^{\circ}$ and are received by a uniform linear array of eight sensors spaced by half-wavelength. The signal at the output of the array is disturbed by calibration errors in addition to the additive noise. These perturbations are assumed uncorrelated Gaussian variables with zero mean. In all simulations, we limit our discussion to small calibration errors and consider the signal to perturbation ratio (SPR) for the values 30, 35, and $40 \mathrm{~dB}$. In the STFD based method, a PWVD with rectangular window length of 129 is applied to the sensor output data. The observation period is 1024 snapshots and the results are averaged over 500 independent Monte Carlo trials. DOA estimates $\tilde{\theta}_{1}$ and $\tilde{\theta}_{2}$ are obtained for each Monte Carlo run by locating the peaks of the spectrum and comparing them to $\theta_{1}$ and $\theta_{2}$, respectively. The variance of the differences $\Delta \theta_{1}=\tilde{\theta}_{1}-\theta_{1}$ and $\Delta \theta_{2}=\tilde{\theta}_{2}-\theta_{2}$, constitute the simulation results. As for numerical results related to the variance terms in Equations (48) and (49), they are obtained by Monte Carlo method.

Figures 1, 2, 3, and 4 show the statistical performance of each method versus the SNR for different values of the SPR. The curves in these figures compare the empirical standard deviation with the theoretical expressions shown by (48) and (49). The results presented in Figures 1 and 3 are obtained using $\Delta \theta_{1}$, whereas the results presented in Figures 2 and 4 are obtained for $\Delta \theta_{2}$. As we can see from Figures 1 and 2, the simulation results for the SOS-based method agree closely with the results of the derived analytical expression for $\mathrm{SNR} \geq 0$ $\mathrm{dB}$. The same observation is made for the STFD-based method from Figures 3 and 4 but for a larger range of SNRs. We can also observe that for the SOS-based method the matching between empirical and theoretical results is lost for very low SNR values (i.e., $\mathrm{SNR}<0$ ) due to the fact that the first order disturbance analysis is not pertinent in this context. However, for the STFD-based method, the matching between empirical and theoretical results still hold because the local SNR value at the auto-source time-frequency point is relatively large even at $\mathrm{SNR}=-10 \mathrm{~dB}$. This robustness with respect to noise may be explained by the effect of spreading the noise power over the time-frequency plan and of localizing the source energy in the $\mathrm{t}-\mathrm{f}$ domain. Moreover, as shown in the above referenced equations, this result is supported by the SNR improvement of a factor equal to the t-f window length $N$ over the SNR associated to SOS based method.

Next, the conducted performance comparison of the SOS and TF-based methods are carried out with respect to the first DOA, the comparison results with respect to the second one being similar. Figure 5 shows no significant difference between the two methods for SNRs $\geq-5$ $\mathrm{dB}$ and high SPRs values, whereas for lower SNRs $(\leq-5$ 


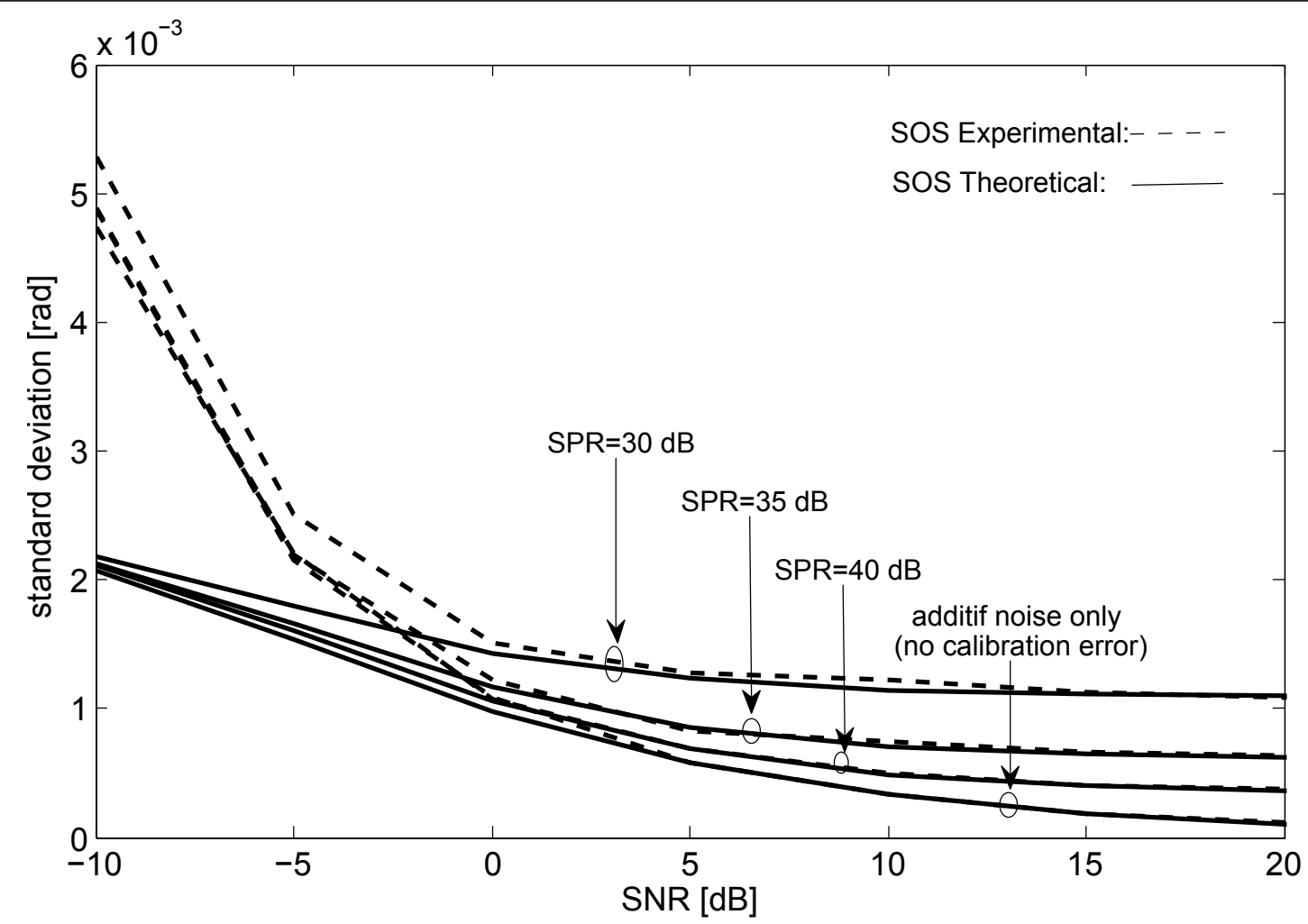

Figure 1 SOS-MUSIC: standard deviation versus SNR for the source at $-10^{\circ}$.

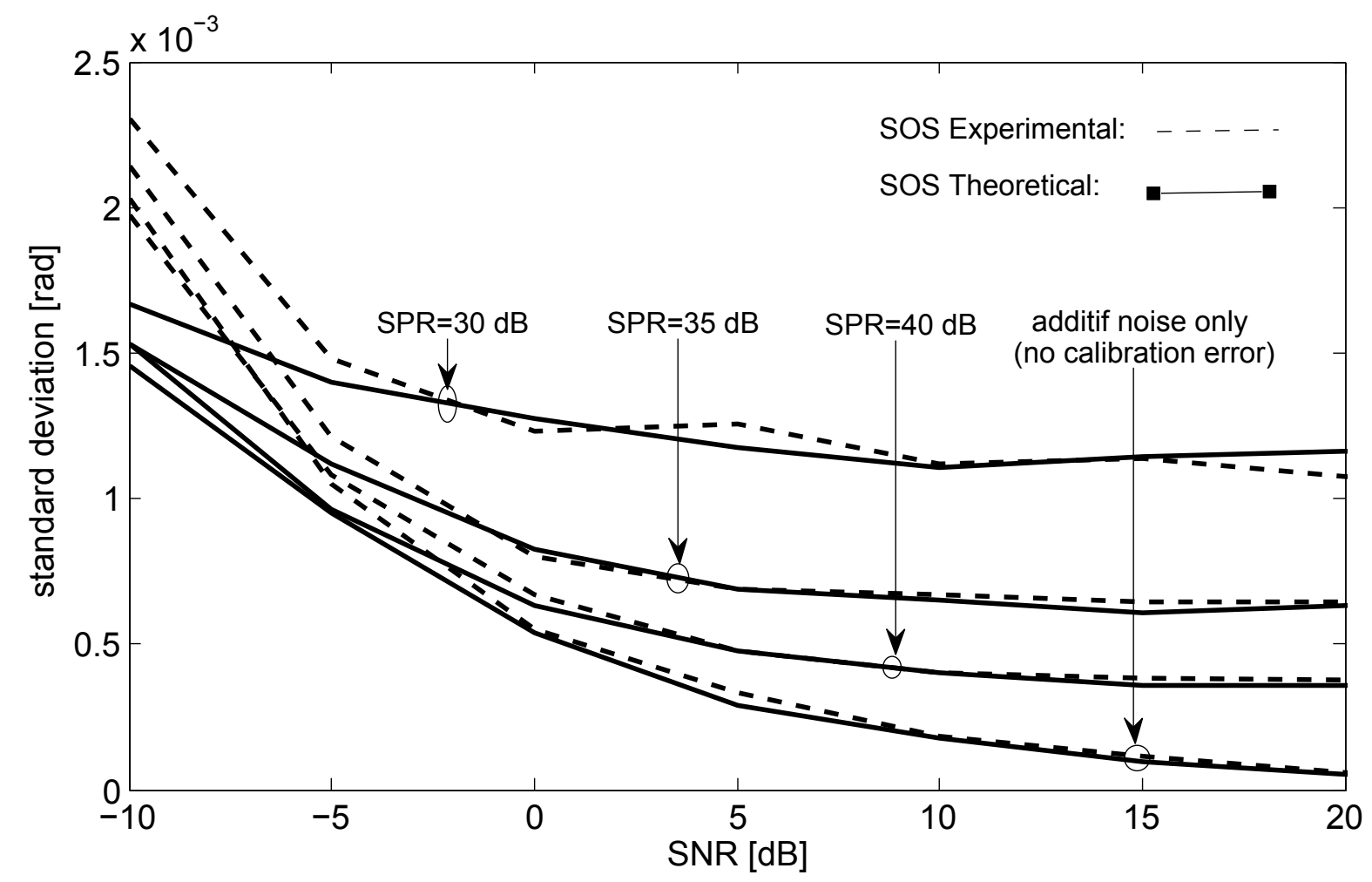

Figure 2 SOS-MUSIC: standard deviation versus SNR for the source at $10^{\circ}$. 


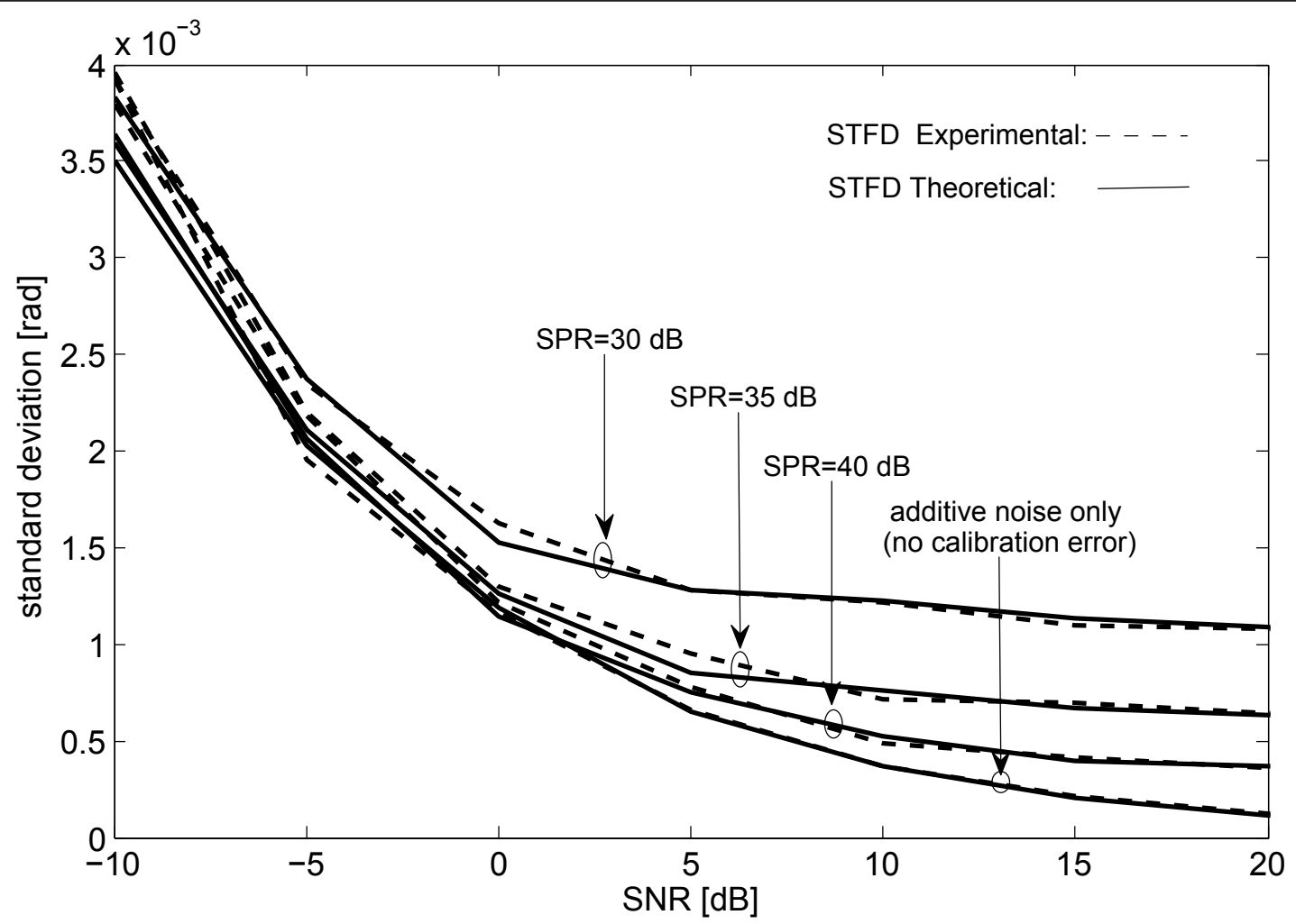

Figure 3 STFD-MUSIC: standard deviation versus SNR for the source at $-10^{\circ}$.

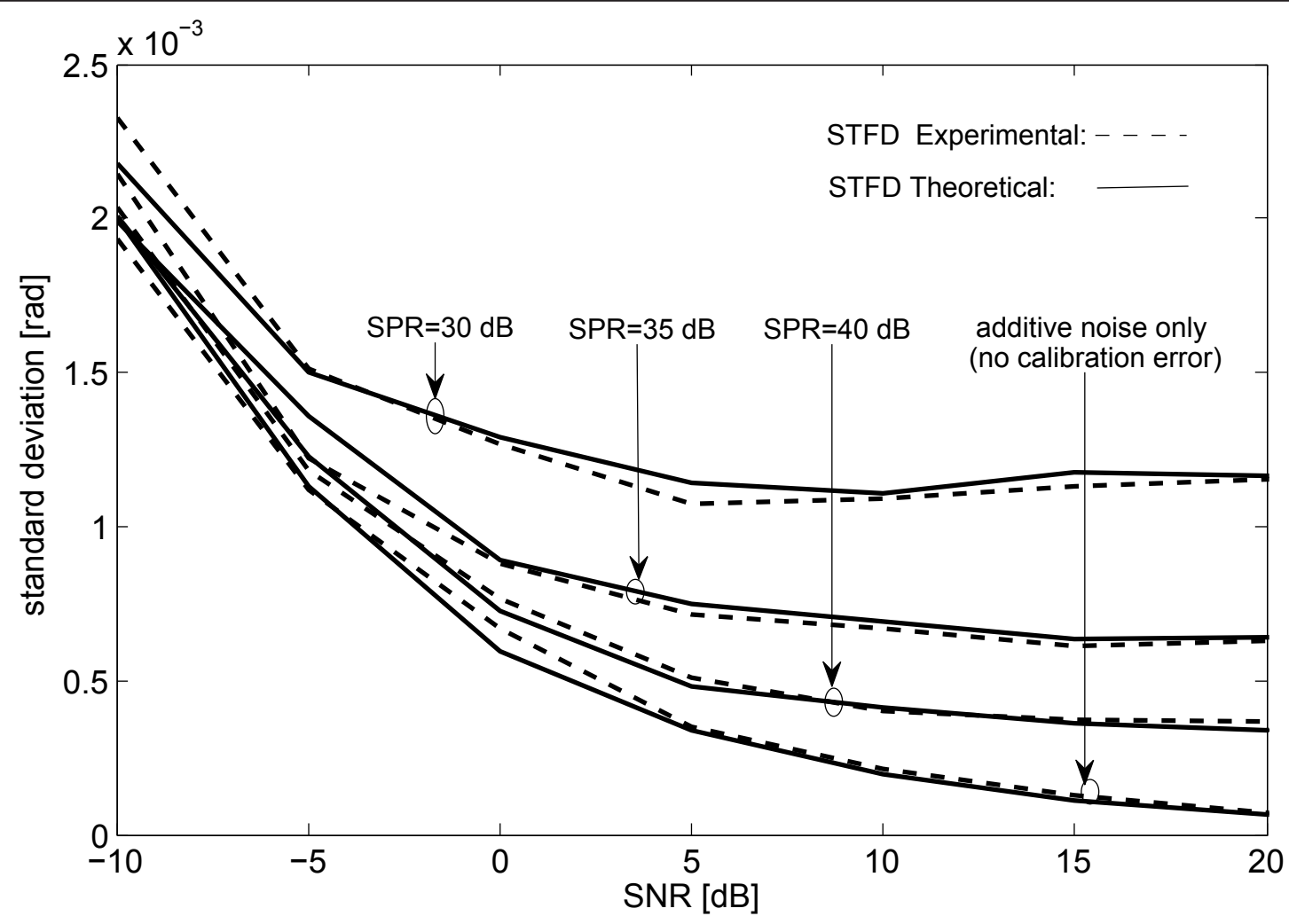

Figure 4 STFD-MUSIC: standard deviation versus SNR for the source at $10^{\circ}$ 


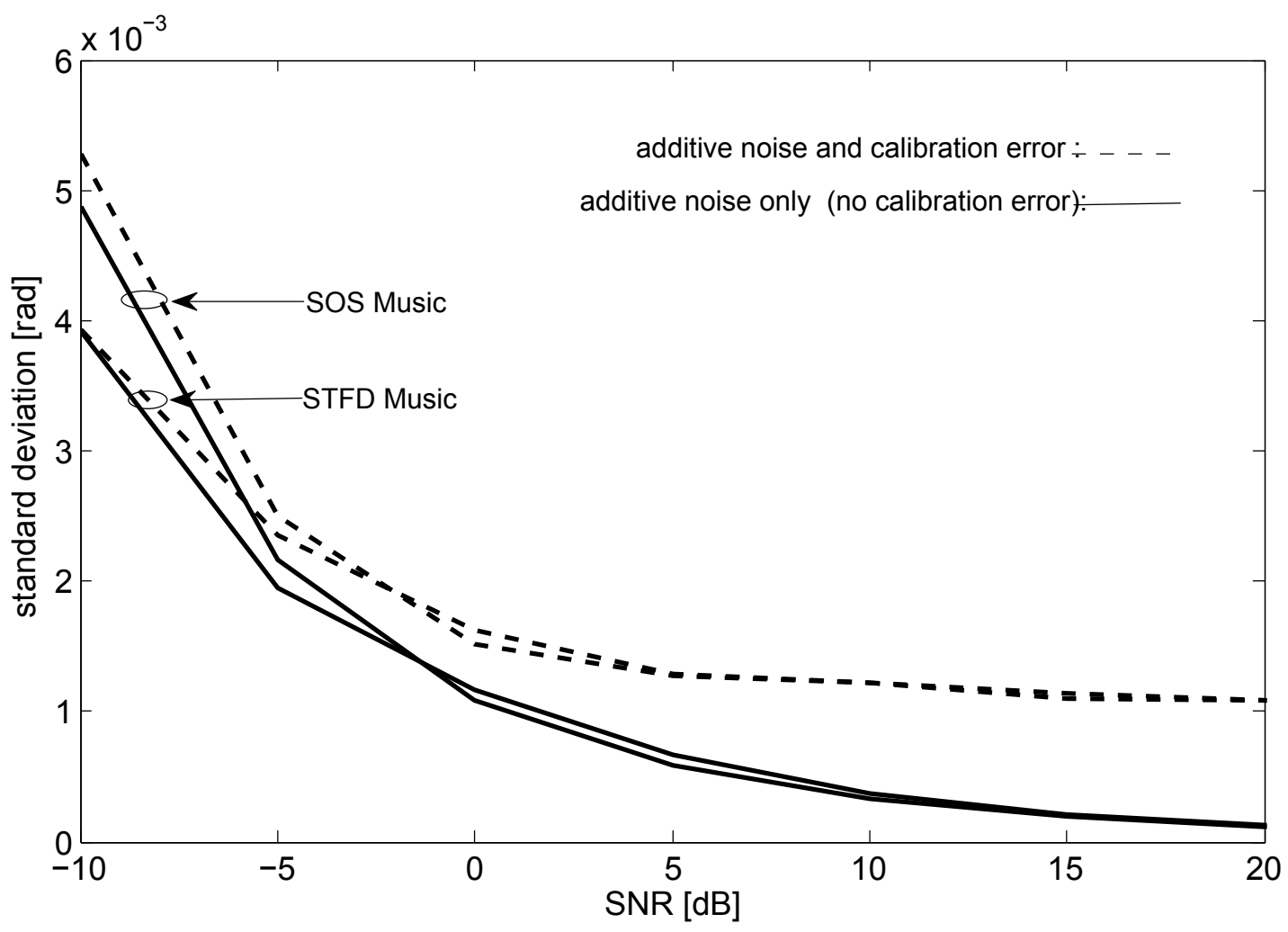

Figure 5 SOS and STFD-MUSIC: standard deviation versus SNR for small calibration errors (SPR $=30 \mathrm{~dB}$ ).

$\mathrm{dB})$ the STFD-based method significantly outperforms the SOS-based one. This difference, as shown in Figures 6 and 7 , is further affected by the number of sensors which is a parameter of resolution and thus of performance. It is clear from these figures that more the number of sensors is large, better is the performance of the algorithms. Figure 6 shows the improvement carried out by the STFD-based method in presence of high additive noise $(\mathrm{SNR}=-10 \mathrm{~dB})$. As expected from data model (4) and Equation (28), it is observed in this figure that the presence of small calibration errors $(\mathrm{SPR}=30 \mathrm{~dB})$ have no significant effect on both methods and all occurs as if there were only the presence of the additive noise. However, as shown in Figure 7, the presence of weak noise with the same calibration error value as in the previous figure, produce a significant performance degradation and the two methods become equivalent.

\section{Conclusion}

The STFD-based direction finding and covariance matrix-based methods have been considered and a unified analytical expression of the DOA error estimation have been derived for both methods. It is shown that in presence of calibration errors and large additive noise the STFD-based method has better performance than the SOS-based method, and that in presence of weak noise both methods are equivalent in their performance. However, even for small sensor perturbations, degradation in performance remains significant because of the multiplicative character of the perturbation with the signal. Through the results obtained in this article, it clearly appears that the TF-MUSIC algorithm plays an important role in the performance improvement, however the implementation of this algorithm may be useless if the sensors are already at the outset too badly calibrated.

\section{Appendix 1: Proof of Equation (11)}

Herein, we derive the expression of (11) given by,

$$
E\left[\Delta \mathbf{A s}(t) \mathbf{s}^{H}(t) \Delta \mathbf{A}^{H}\right]
$$

Before calculate this expression, we start by defining the matrix $\Delta \mathbf{A}$ and the vector $\mathbf{s}(t)$ as,

$$
\Delta \mathbf{A}=\left[\begin{array}{cccc}
\Delta a_{1,1} & \Delta a_{1,2} & \cdots & \Delta a_{1, K} \\
\Delta a_{2,1} & \Delta a_{2,2} & \cdots & \Delta a_{2, K} \\
\vdots & \vdots & \ddots & \vdots \\
\Delta a_{L, 1} & \Delta a_{L, 2} & \cdots & \Delta a_{L, K}
\end{array}\right] \text { and } \mathbf{s}(t)=\left[\begin{array}{c}
s_{1}(t) \\
s_{2}(t) \\
\vdots \\
s_{K}(t)
\end{array}\right]
$$




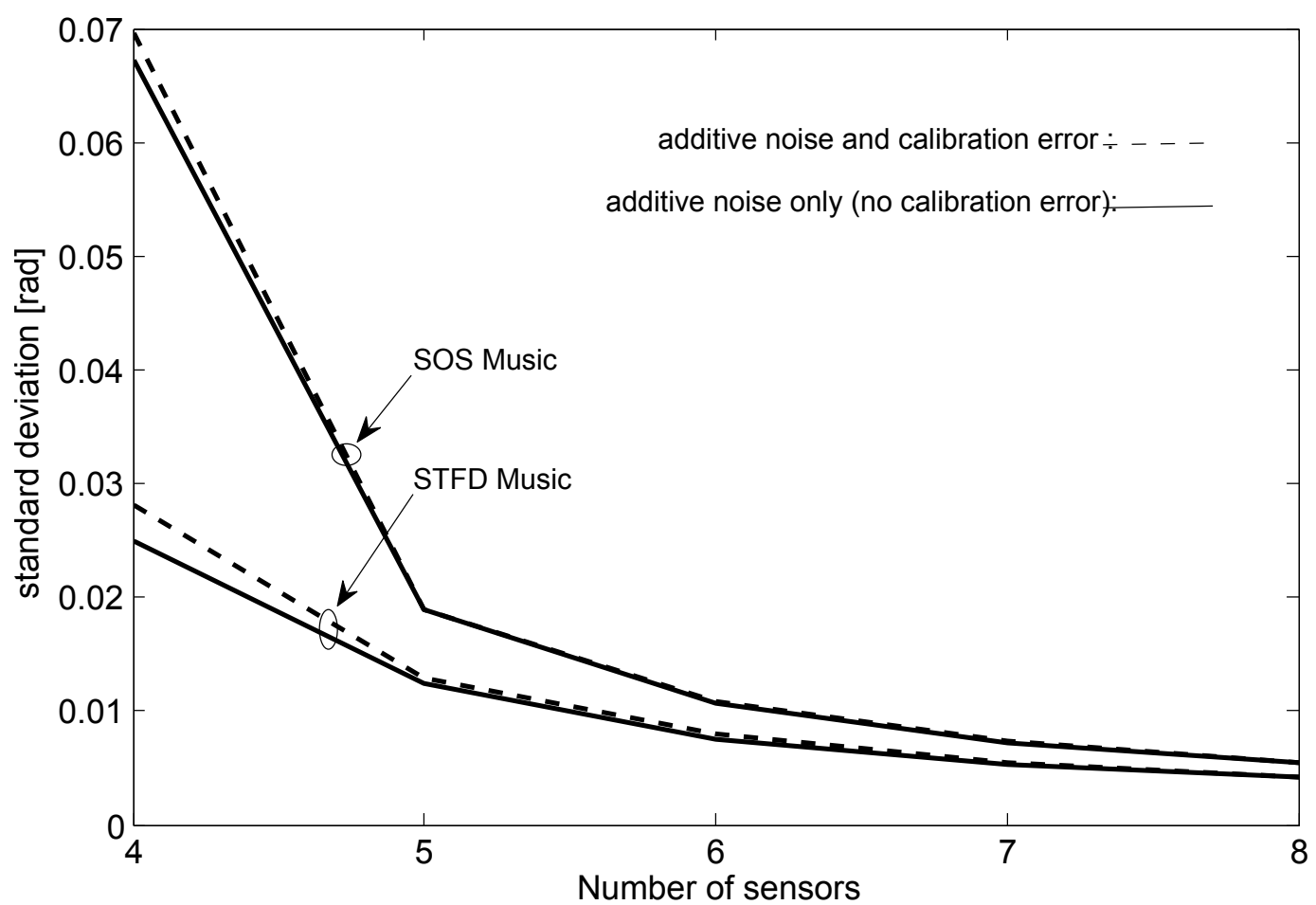

Figure 6 SOS and STFD-MUSIC: standard deviation versus the number of sensors for low SNR $(=-10 \mathrm{~dB})$ and small calibration errors $(\mathrm{SPR}=30 \mathrm{~dB})$.

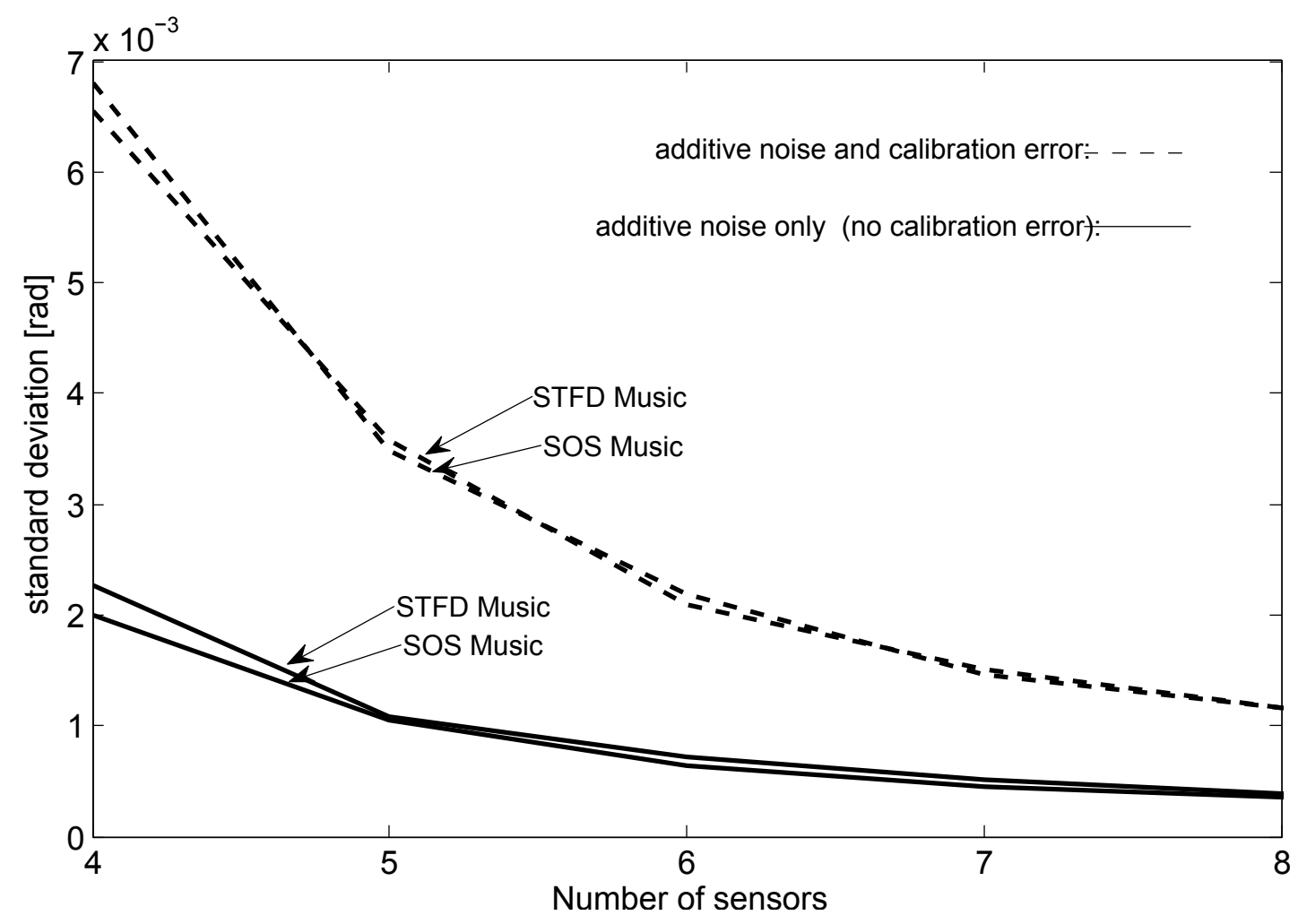

Figure 7 SOS and STFD-MUSIC: standard deviation versus number of sensors for high SNR (= $10 \mathrm{~dB})$ and small calibration errors (SPR $=30 \mathrm{~dB}$ ). 
and omitting the argument $t$ of $s_{k}$ for brevity, we get,

$$
\begin{aligned}
E\left[\Delta \mathbf{A s}(t) \mathbf{s}^{H}(t) \Delta \mathbf{A}^{H}\right] & =E\left\{\left[\sum_{i=1}^{K} \Delta a_{1, i} s_{i} \sum_{i=1}^{K} \Delta a_{2, i} s_{i} \ldots \sum_{i=1}^{K} \Delta a_{L, i} s_{i}\right]^{T}\right. \\
& \left.\times\left[\sum_{j=1}^{K} \Delta a_{1, j}^{*} s_{j}^{*} \sum_{j=1}^{K} \Delta a_{2, j}^{*} s_{j}^{*} \ldots \sum_{j=1}^{K} \Delta a_{L, j}^{*} s_{j}^{*}\right]\right\}
\end{aligned}
$$

Since the source signals $s_{i}, i=1, \ldots, K$, and the sensor errors $\Delta a_{i, j}, j=1, \ldots, L$, are assumed independent and zero mean with variances $\sigma_{s_{i}}^{2}$ and $\sigma_{\Delta a}^{2}$, respectively, the above expression is an $L \times L$ matrix whose elements are given by,

$$
\begin{aligned}
\sigma_{p, q} & =\sum_{i=1}^{K} \sum_{j=1}^{K} E\left[\Delta a_{p, i} \Delta a_{q, j}^{*} s_{i} s_{j}^{*}\right] \\
& =\sum_{i=1}^{K} \sum_{j=1}^{K} E\left[\Delta a_{p, i} \Delta a_{q, j}^{*}\right] E\left[s_{i} s_{j}^{*}\right] \\
& =\sigma_{\Delta a}^{2}\left(\sum_{j=1}^{K} \sigma_{s_{i}}^{2}\right) \delta_{p, q}
\end{aligned}
$$

Therefore, the matrix given by (1.1) is a diagonal matrix which can be expressed as,

$$
E\left[\Delta \mathbf{A s}(t) \mathbf{s}^{H}(t) \Delta \mathbf{A}^{H}\right]=\sigma_{\Delta a}^{2}\left(\sum_{i=1}^{K} \sigma_{s i}^{2}\right) \mathbf{I}=\sigma_{\Delta \mathbf{a}}^{2} \operatorname{Tr}\left(\mathbf{R}_{\mathbf{s s}}\right) \mathbf{I}
$$

\section{Appendix 2: Proof of Equation (25)}

Herein, we derive the expression of (25),

$$
E\left[\overline{\mathbf{D}}_{\tilde{\mathbf{y}} \tilde{\mathbf{y}}}\right]=\frac{1}{K T_{o}} \sum_{k=1}^{K} \sum_{i=1}^{T_{o}} E\left[\mathbf{D}_{\tilde{\mathbf{y}} \tilde{\mathbf{y}}}\left(t_{i}, f_{k, i}\right)\right]
$$

Considering equation (16) for the $k$ th source signal at the $\left(t_{i}, f_{k, i}\right)$ points, and substituting it into (2.1) we have,

$$
E\left[\overline{\mathbf{D}}_{\tilde{y} \tilde{y}}\right]=\frac{1}{K T_{o}} \sum_{k=1}^{K} \sum_{i=1}^{T_{o}}\left\{E\left[\mathbf{a}_{k} d_{s_{k} s_{k}}\left(t_{i}, f_{k, i}\right) \mathbf{a}_{k}^{H}\right]+E\left[\Delta \mathbf{a}_{k} d_{s_{k} s_{k}}\left(t_{i}, f_{k, i}\right) \Delta \mathbf{a}_{k}^{H}\right]+\sigma_{n}^{2} \mathbf{I}\right\}
$$

Taking into account (22) we obtain,

$$
\begin{aligned}
E\left[\overline{\mathbf{D}}_{\tilde{y} \tilde{y}}\right] & =\frac{1}{K T_{o}} \sum_{k=1}^{K}\left\{\sum_{i=1}^{T_{o}} N \sigma_{s_{k}}^{2} \mathbf{a}_{k} \mathbf{a}_{k}^{H}+N \sigma_{s_{k}}^{2} E\left[\Delta \mathbf{a}_{k} \Delta \mathbf{a}_{k}^{H}\right]+\sigma_{n}^{2} \mathbf{I}\right\} \\
& =\frac{N}{K} \sum_{k=1}^{K}\left\{\sigma_{s_{k}}^{2} \mathbf{a}_{k} \mathbf{a}_{k}^{H}+\sigma_{s_{k}}^{2} \sigma_{\Delta a}^{2} \mathbf{I}\right\}+\sigma_{n}^{2} \mathbf{I} \\
& =\frac{N}{K} \sum_{k=1}^{K}\left\{\sigma_{s_{k}}^{2} \mathbf{a}_{k} \mathbf{a}_{k}^{H}\right\}+\frac{N}{K} \sigma_{\Delta a}^{2} \operatorname{Tr}\left(\mathbf{R}_{\mathbf{s s}}\right) \mathbf{I}+\sigma_{n}^{2} \mathbf{I} \\
& =\frac{N}{K} \mathbf{A}(\theta) \mathbf{R}_{\mathbf{s s}} \mathbf{A}^{H}(\theta)+\left[\frac{N}{K} \sigma_{\Delta a}^{2} \operatorname{Tr}\left(\mathbf{R}_{\mathbf{s s}}\right)+\sigma_{n}^{2}\right] \mathbf{I}
\end{aligned}
$$

where $\quad \mathbf{R}_{\mathbf{s s}}=E\left[\mathbf{s}(t) \mathbf{s}^{H}(t)\right]=\operatorname{diag}\left[\sigma_{s_{1}}^{2}, \ldots, \sigma_{s_{K}}^{2}\right]$ and $\operatorname{Tr}\left(\mathbf{R}_{s s}\right)=\left(\sum_{i=1}^{K} \sigma_{s_{i}}^{2}\right)$

\section{Appendix 3: Proof of Equations (33) and (34)}

According to (31) we have,

$$
\mathbf{P}_{\tilde{\mathbf{y}} \tilde{y}}=\tilde{\mathbf{U}}_{s} \tilde{\boldsymbol{\Lambda}}_{s} \tilde{\mathbf{U}}_{s}^{H}+\tilde{\mathbf{U}}_{p} \tilde{\boldsymbol{\Lambda}}_{p} \tilde{\mathbf{U}}_{p}^{H}
$$

Multiplying both sides of $\mathbf{P}_{\tilde{\mathbf{y}} \tilde{\mathbf{y}}}$ by the matrices $\tilde{\mathbf{U}}_{p}^{H}$ and $\tilde{\mathbf{U}}_{s}$ and using the orthogonality property between these matrices, it follows,

$$
\tilde{\mathbf{U}}_{p}^{H} \mathbf{P}_{\tilde{\mathbf{y}} \tilde{\mathbf{y}}} \tilde{\mathbf{U}}_{s}=0
$$

that we can write as,

$$
\left(\mathbf{U}_{p}+\Delta \mathbf{U}_{p}\right)^{H}\left(\mathbf{P}_{\mathbf{y} \mathbf{y}}+\Delta \mathbf{P}_{\mathbf{y y}}\right)\left(\mathbf{U}_{s}+\Delta \mathbf{U}_{s}\right)=0
$$

Expanding the above equation and neglecting the terms higher than the first-order perturbation, and, on another hand, considering relation (27) and the orthogonality property between the signal subspace and the orthogonal subspace, it follows,

$$
\mathbf{U}_{p}^{H} \Delta \mathbf{P}_{\mathbf{y y}} \mathbf{U}_{s}+\Delta \mathbf{U}_{p}^{H} \mathbf{P}_{\mathbf{y} \mathbf{y}} \mathbf{U}_{s}=0
$$

since the eigenvalue decomposition of the perturbation free matrix is given by $\mathbf{P}_{\mathbf{y}}$ 送 $=\mathbf{U}_{s} \Lambda_{s} \mathbf{U}_{s}^{H}$, and using the unitary property of $\mathbf{U}_{s}$ (i.e., $\mathbf{U}_{s}^{H} \mathbf{U}_{s}=\mathbf{I}$ ), we get,

$$
\Delta \mathbf{U}_{p}^{H}=-\mathbf{U}_{p}^{H} \Delta \mathbf{P}_{\mathbf{y y}} \mathbf{U}_{s}\left[\mathbf{U}_{s} \Lambda_{s}\right]^{\#}
$$

where [.] $]^{\#}$ defines the pseudo-inverse operator, hence $\left[\mathbf{U}_{s} \Lambda_{s}\right]^{\#}$ is given by,

$$
\begin{aligned}
{\left[\mathbf{U}_{s} \Lambda_{s}\right]^{\#} } & =\left[\left(\mathbf{U}_{s} \Lambda_{s}\right)^{H}\left(\mathbf{U}_{s} \Lambda_{s}\right)\right]^{-1}\left(\mathbf{U}_{s} \Lambda_{s}\right)^{H} \\
& =\Lambda_{s}^{-1} \mathbf{U}_{s}^{H}
\end{aligned}
$$

Substituting (3.6) in (3.5), we obtain,

$$
\Delta \mathbf{U}_{p}=-\mathbf{U}_{s} \Lambda_{s}^{-1} \mathbf{U}_{s}^{H} \Delta \mathbf{P}_{\mathbf{y y}}^{H} \mathbf{U}_{p}
$$

Using the same steps applied to derive the above equation, we obtain the expression of the signal subspace perturbation $\Delta \mathbf{U}_{s}$ versus the perturbation matrix $\Delta \mathbf{P}_{\mathbf{y y}}$

$$
\Delta \mathbf{U}_{s}=\mathbf{U}_{p} \mathbf{U}_{p}^{H} \Delta \mathbf{P}_{\mathbf{y y}} \mathbf{U}_{s} \Lambda_{s}^{-1}
$$




\section{Appendix 4: Proof of Equation (40)}

Herein, we derive the expression (40) of the estimate of the error $\Delta \theta_{k}, k=1, \ldots, K$, due to the sensor error and the additive noise. For this purpose, we use the extremum search method which consists of finding the zeros of the derivative of the objective function derived from the estimated orthogonal subspace,

$$
F(\theta)=\mathbf{a}(\theta){ }^{H} \tilde{\mathbf{U}}_{p} \tilde{\mathbf{U}}_{p}^{H} \mathbf{a}(\theta)
$$

A well known method is to expand under Taylor series the first partial derivative of the above function with respect to $\theta$ and to use a second-order approximation to extract $\Delta \theta_{k}$,

$$
\begin{aligned}
\frac{\partial F\left(\tilde{\theta}_{k}\right)}{\partial \theta} & =\sum_{n=0}^{\infty} \frac{1}{n !} \frac{\partial^{n+1} F\left(\theta_{k}\right)}{\partial \theta^{n+1}\left(\Delta \theta_{k}\right)^{n}} \\
& =\frac{\partial F\left(\theta_{k}\right)}{\partial \theta}+\frac{\partial^{2} F\left(\theta_{k}\right)}{\partial \theta^{2}} \Delta \theta_{k}+\ldots+\frac{1}{i !} \frac{\partial^{i+1} F\left(\theta_{k}\right)}{\partial \theta^{i+1}} \Delta \theta_{k}^{i}+\ldots,
\end{aligned}
$$

that we approximate by the two first terms,

$$
\frac{\partial F\left(\tilde{\theta}_{k}\right)}{\partial \theta} \approx \frac{\partial F\left(\theta_{k}\right)}{\partial \theta}+\frac{\partial^{2} F\left(\theta_{k}\right)}{\partial \theta^{2}} \Delta \theta_{k}
$$

Setting the above equation to zero leads to the following expression of $\Delta \theta_{k}$,

$$
\Delta \theta_{k} \approx-\frac{\frac{\partial F\left(\theta_{k}\right)}{\partial \theta}}{\frac{\partial^{2} F\left(\theta_{k}\right)}{\partial \theta^{2}}}, \quad k=1, \ldots, K
$$

where,

$$
\frac{\partial F\left(\theta_{k}\right)}{\partial \theta_{k}}=\mathbf{a}^{(1)}\left(\theta_{k}\right)^{H} \tilde{\mathbf{U}}_{p} \tilde{\mathbf{U}}_{p}^{H} \mathbf{a}\left(\theta_{k}\right)+\mathbf{a}\left(\theta_{k}\right)^{H} \tilde{\mathbf{U}}_{p} \tilde{\mathbf{U}}_{p}^{H} \mathbf{a}^{(1)}\left(\theta_{k}\right)
$$

and,

$$
\frac{\partial^{2} F\left(\theta_{k}\right)}{\partial \theta_{k}^{2}}=\mathbf{a}^{(2)}\left(\theta_{k}\right)^{H} \tilde{\mathbf{u}}_{p} \tilde{u}_{p}^{H} \mathbf{a}\left(\theta_{k}\right)+2 \mathbf{a}^{(1)}\left(\theta_{k}\right) \tilde{\mathbf{u}}_{p} \tilde{u}_{p}^{H} \mathbf{a}^{(1)}\left(\theta_{k}\right)+\mathbf{a}\left(\theta_{k}\right)^{H} \tilde{\mathbf{u}}_{p} \tilde{u}_{p}^{H} \mathbf{a}^{(2)}\left(\theta_{k}\right),
$$

the superscripts (1) and (2) correspond to the first and the second-order derivatives of $\mathbf{a}(\theta)$ with respect to $\theta$, respectively.

Substituting $\tilde{\mathbf{U}}_{p}=\mathbf{U}_{p}+\Delta \mathbf{U}_{p}$ in the above equations, and using the property of orthogonality between the subspaces spanned by $\mathbf{a}\left(\theta_{k}\right)$ and $\mathbf{U}_{p}$ (i.e., $\mathbf{a}\left(\theta_{k}\right)^{H} \mathbf{U}_{p}=0$ ), and neglecting the derivatives and the perturbation terms of second-order, we obtain,

$$
\frac{\partial F\left(\theta_{k}\right)}{\partial \theta_{k}}=2 \operatorname{Re}\left[\mathbf{a}\left(\theta_{k}\right)^{H} \Delta \mathbf{U}_{p} \mathbf{U}_{p}^{H} \mathbf{a}^{(1)}\left(\theta_{k}\right)\right]
$$

and

$$
\frac{\partial^{2} F\left(\theta_{k}\right)}{\partial \theta_{k}^{2}}=2 \mathbf{a}^{(1)}\left(\theta_{k}\right)^{H} \mathbf{U}_{p} \mathbf{U}_{p}^{H} \mathbf{a}^{(1)}\left(\theta_{k}\right)+2 \operatorname{Re}\left[\mathbf{a}^{(1)}\left(\theta_{k}\right)^{H} \mathbf{U}_{p} \Delta \mathbf{U}_{p}^{H} \mathbf{a}^{(1)}\left(\theta_{k}\right)\right]
$$

Assuming that $\Delta \mathbf{U}_{p}=\varepsilon \mathbf{U}_{p}$, where $\varepsilon \ll 1$, the above equation can be written as,

$$
\begin{aligned}
\frac{\partial^{2} F\left(\theta_{k}\right)}{\partial \theta_{k}^{2}} & =2(1+\varepsilon)\left\|\mathbf{a}^{(1)}\left(\theta_{k}\right)^{H} \mathbf{U}_{p}\right\|^{2} \\
& \approx 2\left\|\mathbf{a}^{(1)}\left(\theta_{k}\right)^{H} \mathbf{U}_{p}\right\|^{2}
\end{aligned}
$$

Substituting (4.7) and (4.9) into (4.4), it results the expression of the perturbation in the $k$ th estimated direction of arrival as follows,

$$
\Delta \theta_{k}=\frac{\operatorname{Re}\left[-\mathbf{a}\left(\theta_{k}\right)^{H} \Delta \mathbf{U}_{p} \mathbf{U}_{p}^{H} \mathbf{a}^{(1)}\left(\theta_{k}\right)\right]}{\left\|\mathbf{a}^{(1)}\left(\theta_{k}\right)^{H} \mathbf{U}_{p}\right\|^{2}}
$$

\section{Author details}

${ }^{1}$ Electrical Engineering Department, Ecole Nationale Polytechnique, Algiers, Algeria ${ }^{2}$ Télécom ParisTech, TSI, 75634, Paris Cedex 13, France

\section{Competing interests}

The authors declare that they have no competing interests.

\section{Received: 17 October 2011 Accepted: 30 April 2012}

Published: 30 April 2012

\section{References}

1. F Li, H Liu, RJ Vaccaro, Performance analysis for DOA estimation algorithms: unification, simplification, and observations. IEEE Trans. Aerosp. Electron. Syst. 29(4), 1170-1183 (1993)

2. P Stoica, A Nehorai, MUSIC, Maximum Likelihood, and Cramer-Rao Bound. IEEE Trans. Acoustics Speech Signal Processing. 37(5), 720-741 (1989)

3. A Belouchrani, M Amin, Time-frequency MUSIC. IEEE Signal Process. Lett. 6 , 109-110 (1999)

4. A Belouchrani, M Amin, A new approach for blind source separation using time-frequency distribution, in Proceeding SPIE Conference on Advanced Algorithms and Architectures for Signal Processing, vol. 2846. Denver, Colorado, pp. 193-203 (Oct 1996)

5. B Belouchrani, M Amin, Blind source separation based on time-frequency signal representations. IEEE Trans. Signal Process. 46(11), 2888-2898 (1998)

6. B Boashash, Time-Frequency Signal Analysis and Processing: A Comprehensive Reference, (Elsevier Science Publ, San Diego, 2003)

7. Z Yimin, M Weifeng, M Amin, Subspace analysis of spatial time-frequency distribution matrices. IEEE Trans. Signal Process. 49(4), 747-759 (2001)

8. F Li, RJ Vaccaro, Sensitivity analysis of DOA estimation algorithms to sensor errors. IEEE Trans. Aerosp. Electron. Syst. 28(3), 708-717 (1992)

9. AL Swindlehurst, T Kailath, A performance analysis of subspace-based methods in the presence of model errors, Part I: the MUSIC algorithm. IEEE Trans. Signal Process. 40(7), 1758-1774 (1992)

10. AL Swindlehurst, A maximum a posteriori approach to beamforming in the presence of calibration errors, in Proc. 8th IEEE Workshop Stat. Signal Array Process, Corfu, Greece, pp. 82-85 (June 1996)

11. J Yang, AL Swindlehurst, The effect of array calibration errors on DF-based signal copy performance. IEEE Trans. Signal Process. 43(11), 2724-2732 (1995)

12. C Vaidyanathan, KM Buckley, Performance analysis of the MVDR spatial spectrum estimator. IEEE Trans. Signal Process. 43(6), 1427-1437 (1995)

doi:10.1186/1687-6180-2012-94

Cite this article as: Khodja et al.: Performance analysis for timefrequency MUSIC algorithm in presence of both additive noise and array calibration errors. EURASIP Journal on Advances in Signal Processing 2012 2012:94. 